\title{
MD Study on Carbon Sputtering and Redeposition
}

\author{
By Tetsuya MURAmoto ${ }^{1)}$ and Toru HYAKUTAKE ${ }^{2)}$ \\ 1) Okayama University of Science, Okayama, Japan \\ 2) Yokohama National University, Kanagawa, Japan
}

(Received June 21st, 2011)

\begin{abstract}
In order to estimate the erosion of accelerator grids of an ion engine, the low-energy sputtering of carbon material under $\mathrm{Xe}$ ion bombardment and the redeposition of low-energy sputtered particles on the carbon material are studied through the molecular dynamics (MD) simulation. For the normal incidence of Xe, the MD result of sputtering yield almost agrees with the experimental result by Williams et al. (AIAA-2004-3788). However, the experimental result shows less incident angle dependence than the MD result because it performed on a rough surface. For the redeposition on amorphous carbon (a-C) surface, the reflection and the deep trapping are suppressed. The mean deposition rate with deposition energy up to $100 \mathrm{eV}$ is $0.82 \sim 0.75$ taking account of incident angle distributions.
\end{abstract}

Key Words: Molecular Dynamics Simulation, Low-Energy Sputtering, Sputtering Deposition

\section{Introduction}

Ion engine had been developed by JAXA for a long-term space transportation, such as the Hayabusa probe, with minimum propellant. The ion engine has a lifetime that is dominated by the deformation of the carbon accelerator grid in $\mathrm{Xe}$ ion flow. Low-energy sputtering and redeposition of sputtered particles bring the deformation of the carbon device. At present those knowledge are being researched to evaluate the ion engine lifetime ${ }^{1)}$. MD simulation is one of the most powerful methods for the investigation of the low-energy sputtering and the redeposition, because it can treat a wide range of interaction energies and the diversity of $\mathrm{C}-\mathrm{C}$ bonding.

In this paper, we summarize the MD study on the low-energy sputtering ${ }^{2)}$ and the redeposition ${ }^{3)}$ in order to develop the ion engine lifetime evaluation code.

\section{Simulation Model}

Molecular dynamics can be described as the computation of the motion of systems of particles from knowledge of the interaction forces between particles ${ }^{4)}$. The dynamics of the system can be determined by solving Newton's laws of motion for each particle. In the present MD simulation, the C-C force for an equilibrium range is described by the analytical bond-order (BO) potential ${ }^{5)}$ which reproduces the property of the carbon crystal, the dangling bond, $\mathrm{sp}^{1}, \mathrm{sp}^{2}$ and $\mathrm{sp}^{3}$ bonding. The $\mathrm{C}-\mathrm{C}$ force in a short range is treated by Molière potential ${ }^{6}$ ) which is one of the screened Coulomb potentials. The pair repulsion term of the $\mathrm{BO}$ potential is smoothly connected to the Molière potential using a cubic interpolating function, and the cohesive term of the BO potential is smoothly attenuated in a short range using a cubic cut-off function. The $\mathrm{Xe}-\mathrm{C}$ force is calculated by the Lennard-Jones ${ }^{7)}$ and Molière potentials, which are smoothly connected in a similar manner to the C-C force.

The accelerator grid of ion engine is structured by carbon composite (CC) which is the graphite fiber assemblage with the density of $1.7 \sim 1.9 \mathrm{~g} / \mathrm{cm}^{3}$. However the surface bombarded by the low-energy Xe ion in this work is employed a diamond crystalline to simplicity of the substrate modeling. The crystalline is randomly oriented, and it is formed into a flat surface. This surface is similar to a diamond polycrystalline in microscopic view. The substrate diameter is $25 \sim 36 \AA$ which is a minimum size that can include the collision cascade.

In the redeposition simulation, the surface is set into an a-C in order to treat the $\mathrm{C}-\mathrm{C}$ bond formation in the low-energy range. The RMS roughness of the a-C surface is $1.5 \AA$. The mean density of the a-C bulk is $2.8 \mathrm{~g} / \mathrm{cm}^{3}$, and the mean fraction of $\mathrm{sp}^{2}$ and $\mathrm{sp}^{3}$ bonding is $77 \%$ and $21 \%$, respectively. The a-C surface has low density and some unstable bonding. This is prepared through the MD simulation of carbon film growth under the serial incidence of carbon particles. In order to reconstruct the sputter-deposited surface, we employed the Thompson formula ${ }^{8)}$ as the typical sputtering deposition energy distribution. The cycle of deposition is $5 \mathrm{ps}$, and 12 monolayer is deposited. This preparation is similar to MD work of Cooper et al. ${ }^{9)}$. The substrate has a periodic horizontal boundary. The boundary size is $36 \AA \times 36 \AA$, and the thickness of substrate is $12 \AA$.

In order to be the substrate in a part of larger material, a damped boundary ${ }^{10)}$ and a heat-bath region are laid in the bottom of the substrate, the temperature of which is controlled around $300 \mathrm{~K}$ using the Langevin MD method ${ }^{11)}$.

\section{Results and Discussions}

\subsection{Low-energy Xe bombardment on carbon}

A number of Monte Carlo codes (e.g. ACAT ${ }^{12)}$, TRIM.SP ${ }^{13)}$ ) based on binary-collision approximation (BCA) have been developed to analyze ion-solid interactions. For high-energy ions, these are used successfully to predict sputtering yield, ion ranges, angular and energy distribution of backscattered ions and sputtered particles. However, the BCA breaks down at low energies when many-body effects become important.

In Fig. 1, we show the incident energy dependence of the 


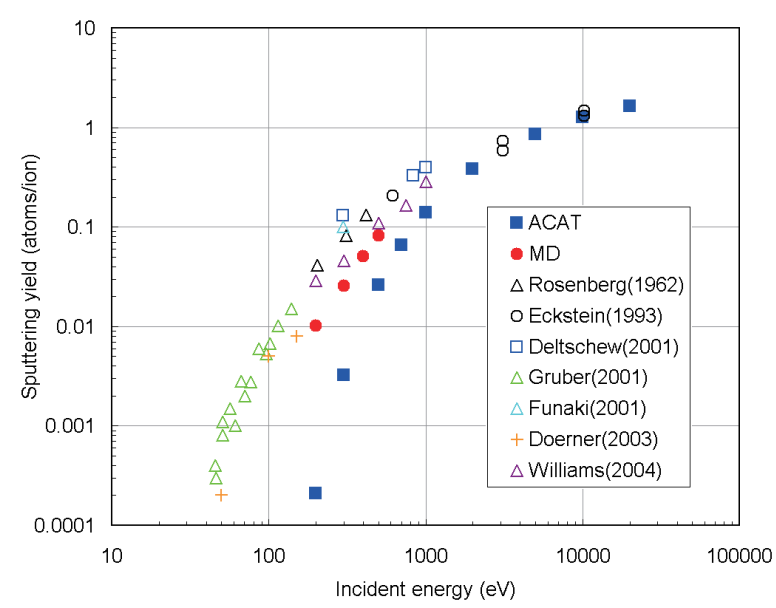

Fig. 1. Sputtering yield of carbon irradiated by Xe ion at $\alpha=0^{\circ}$.

sputtering yield at normal incidence. Available calculation ${ }^{14)}$ and experimental results ${ }^{15-20)}$ are plotted to compare. The ACAT calculation underestimates by $10^{-2}$ than the experimental result at $200 \mathrm{eV}$. However the MD calculation almost agrees with the experimental result. The enhancement of the sputtering yield is caused by the high-density energy deposition on the surface. It is produced by the many-body collision in the case of the low-energy sputtering ${ }^{21)}$. On the other hand, it suggested that the Xe retention on the surface bring on the high-density energy deposition ${ }^{22}$. The microscopic surface analysis of the accelerator grid is demanded to identify the dominant factor between the many-body collision and the Xe retention.

The microscopic surface structure is one of the most important factors for the sputtering phenomena. Since the oblique incidence has a long interaction time to the surface, the surface topology is strongly influence to the sputtering yield. Figure 2 shows the incident angle dependence of the sputtering yield at the incident energy of $200 \mathrm{eV}$. The incident angle $\alpha$ is measured from the surface normal. The MD calculation overestimates by $10^{1}$ than the experimental result ${ }^{15)}$ at $\alpha=70^{\circ}$. It seems that the density of diamond crystalline in the MD calculation is lager than the density of $\mathrm{CC}$ in the experiment. The surface analysis after Xe irradiation in the atomistic view, eg. Atomic Force Microscope, Rutherford Backscattering Spectrometry and X-ray Photoelectron

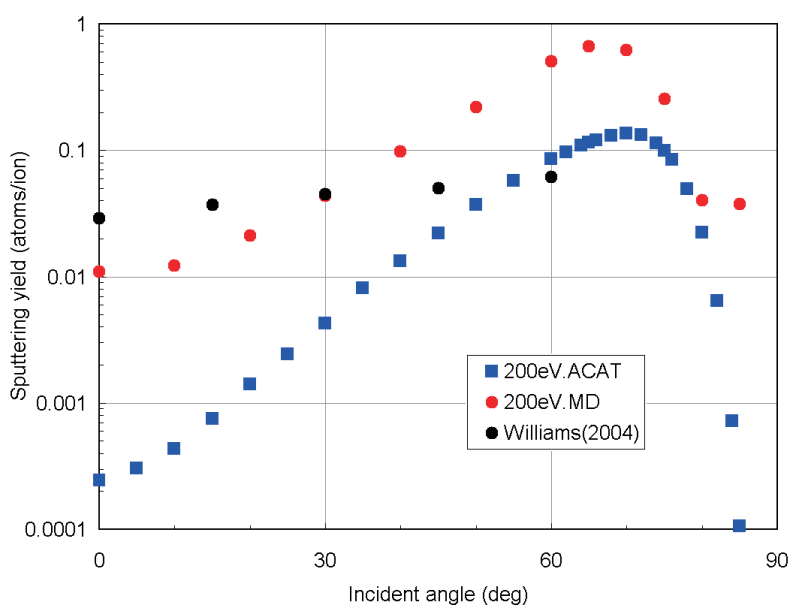

Fig. 2. Sputtering yield of carbon irradiated by $200 \mathrm{eV} \mathrm{Xe}$ ion.

Spectroscopy, is demanded to estimate the realistic sputtering phenomenon.

The experimental result has a less incident angle dependence than the simulation result. In the experiment, the CC surface has probably a mesoscopic surface roughness. When a low-energy projectile approach to the surface, the many-body collision is caused to a group of surface atoms and the projectile feels the direction of the local surface normal. Thus, the randomization of the surface normal is important in the low-energy sputtering, and it results in the less incident angle dependence in the experiment.

The MD simulation can not treat the mesoscopic surface topology from the limitation of the computer performance. However if it is approximated by a group of microscopic flat surfaces, the sputtering of the mesoscopic rough surface can be reconstructed by the Monte-Carlo simulation based on the sputtering database of this work as shown in Fig. 3.

In order to estimate the redeposition, one should know the velocity distribution of sputtered particles. Form the information of sputtered particles in the MD simulation, we defined the differential sputtering yield as following,

$$
\frac{d Y}{d \Omega}=Y_{\text {total }}(E, \alpha) f(\theta, \varphi)
$$

where $Y_{\text {total }}$ is the sputtering yield (atom/ion), $E$ is the incident energy of Xe ions, $\alpha$ is the incident angle, $f$ is the normalized differential sputtering yield (1/sr), $\theta$ and $\varphi$ are the polar and

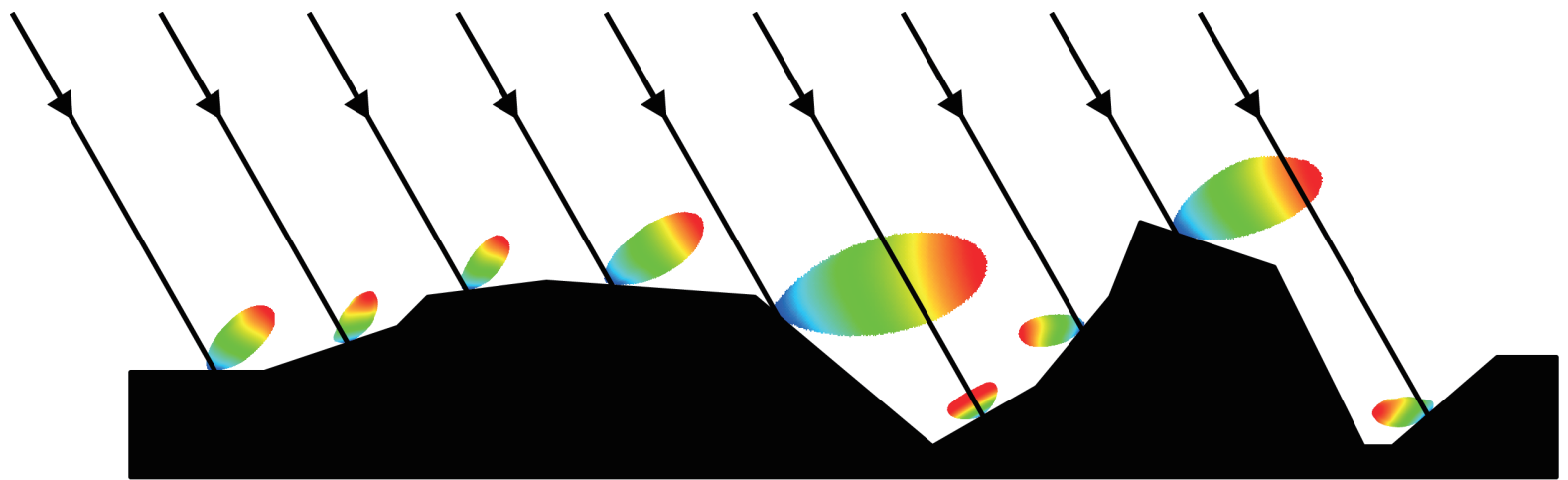

Fig. 3. Modeling of mesoscopic rough surface sputtering. 


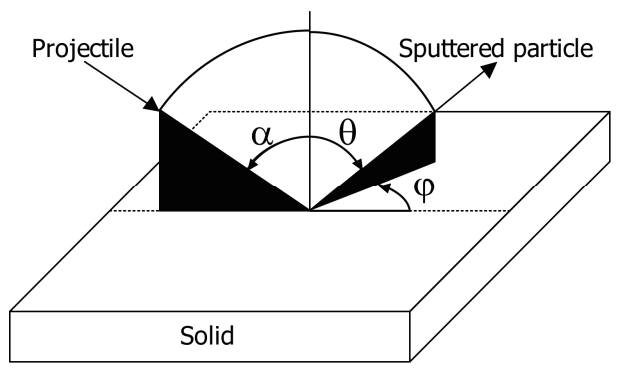

Fig. 4. Geometry of incident angle and ejection angle.

azimuth angle of the ejecting direction, respectively. We show the geometry in Fig. 4.

In Fig. 5, $f(\theta, \varphi)$ for $200 \mathrm{eV}$ Xe incident is shown in contour plots. The radial and axial scale indicates $\theta=0^{\circ} \sim 90^{\circ}$ and $\varphi=$ $0^{\circ} \sim 360^{\circ}$, respectively. The curves of nine colors from blue to red are corresponding to the contour lines of 10, 20, 30, 40, 50, $60,70,80$ and $90 \%$ of the maximum value of $f(\theta, \varphi)$. At $\alpha=0^{\circ}$, $f(\theta, \varphi)$ is a hart shape, and $f(\theta, \varphi)$ at $\alpha=60^{\circ}$ has a peak at a direction along the incident direction. This feature means that sputtered particles has memory of the projectile because the sputtered particles by the low-energy projectile undergo only a few collisions to the ejection.

\subsection{Low-energy redeposition of carbon atom}

Carbon forms a great variety of crystalline and disordered structures because it is able to exist in three hybridizations, $\mathrm{sp}^{3}$, $\mathrm{sp}^{2}$ and $\mathrm{sp}^{1}$. From the potential energy of deposited atoms, we can sort its trapping state in coordination 1, coordination 2 ( $\mathrm{sp}^{1}$ bonding), and coordination 3 and 4 ( $\mathrm{sp}^{2}$ and $\mathrm{sp}^{3}$ bonding). The coordination 1 and 2 corresponds to an unstable bonding in a shallow trap, and the coordination 3 and 4 is a metastable bonding in a deep trap.

In Fig. 6, reflection and trapping ratio maps are shown as a function of $E$ and $\alpha$. In order to compare with the a-C surface, the (111) diamond surface is also considered. The ratio is indicated in gray scale, where areas in five colors from white to black indicate the ratio of $0 \sim 20 \%, 20 \sim 40 \%, 40 \sim 60 \%$, $60 \sim 80 \%$ and $80 \sim 100 \%$. For the (111) surface, frequent

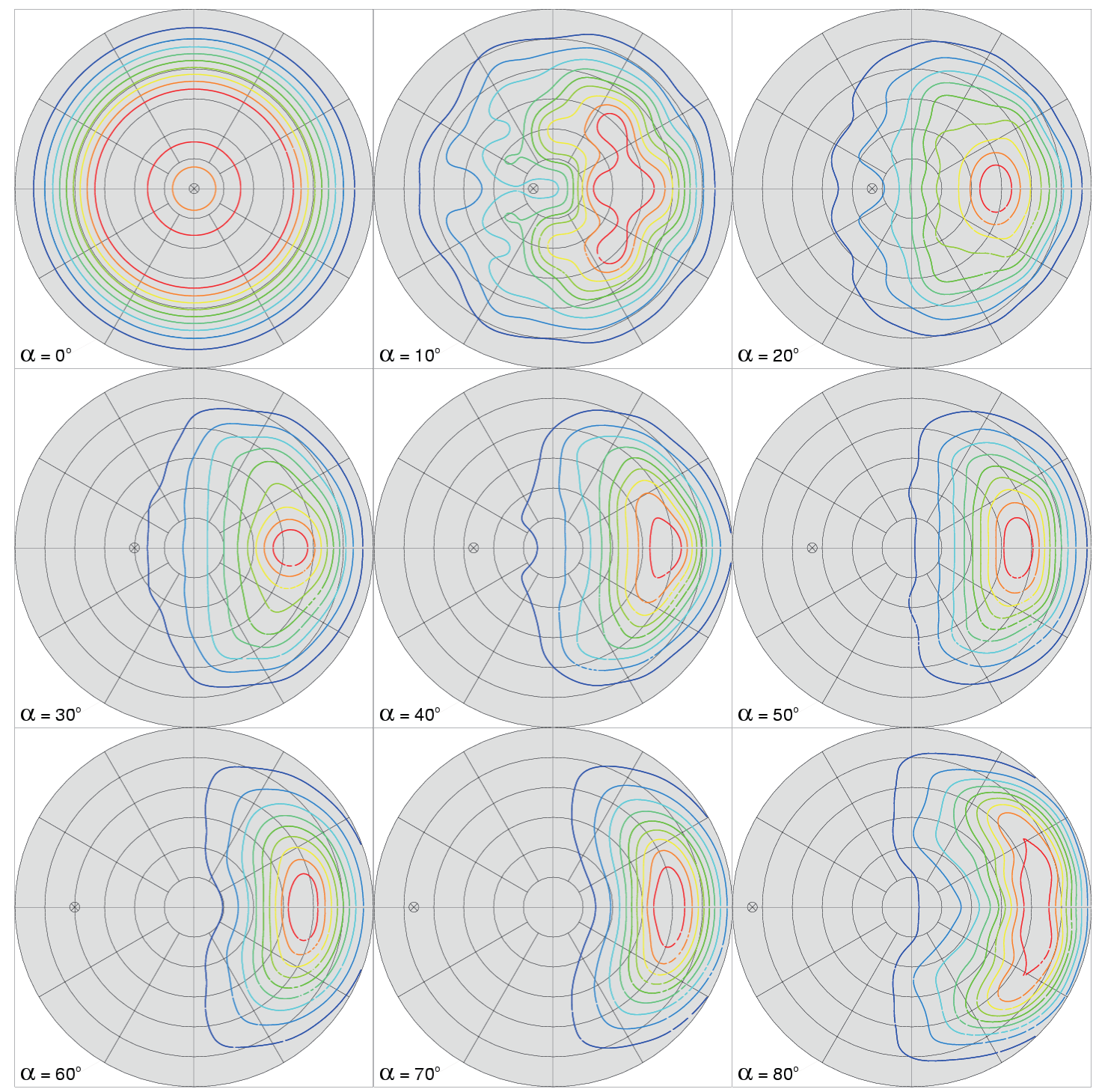

Fig. 5. Contour plot of differential sputtering yield of carbon irradiated by $200 \mathrm{eV} \mathrm{Xe}$ ion. 
shallow trapping appears at $E \leq 20 \mathrm{eV}$ because the deposited atom makes a soft landing. The deep trapping occurs frequently in $E \geq 30 \mathrm{eV}$ about normal incidence because the deposition energy is enough to penetrate the top layer of (111) surface for the subsurface implantation. A high reflection rate appears in $E \geq 30 \mathrm{eV}$ about grazing incidence because the energy of horizontal motion is too large to make $\mathrm{C}-\mathrm{C}$ bond on surface.

For the a-C surface, the reflection and the deep trapping ratio are less than that on the (111) surface. Since the a-C surface is low density and low coordination, the deposited atom hardly causes collisions above the surface for the reflection, and is more captured into the shallow trap in the surface. As a result, the shallow trapping ratio is more than that on the (111) surface.

In Fig. 7, we show the sputtering yield map as a function of $E$ and $\alpha$. The yield per incident atom is indicated in gray scale, where areas in six colors from white to black indicate the ratio of $0 \sim 0.1,0.1 \sim 0.2,0.2 \sim 0.3,0.3 \sim 0.4,0.4 \sim 0.5$ and $0.5 \sim 0.6$. For the (111) surface, the sputtering appears in $E>80 \mathrm{eV}$ and $\alpha=$ $30^{\circ} \sim 50^{\circ}$. The effective energy deposition in the surface is possible in oblique incidence with less reflection.

On the other hand, the sputtering yield on the a-C is especially large about grazing incidence. Since the reflection on the a-C is suppressed, the grazing incidence causes the effective energy deposition. Moreover, some sputtering is caused even with $E=10 \mathrm{eV}$ although the deposition energy is not enough to a kinetic sputtering process. The sputtering yield per incident particle is $0.004 \sim 0.055$ at $E=10 \mathrm{eV}$. This result means that the sputtering correlates to the contact of the deposited particle and the surface. For example, some unstable bonds in the a-C surface are broken by the influence of the contact, and then particles take off from the breaking bond. Therefore this is most likely a chemical process.

In this section, the reflection rate and the sputtering yield have been discriminated. The sum of these is the lost atom per incident atom and is called the self-sputtering yield $Y_{\text {self. Thus }}$ the deposition rate is the weighted average of $1-Y_{\text {self }}$ over the velocity distribution of deposited particles. We take into account the following distribution:

$$
y(E, \alpha) \mathrm{d} E \mathrm{~d} \Omega=F(E) \mathrm{dE} g(\alpha) \mathrm{d} \Omega,
$$

where $F(E)$ is an approximation of the Thompson formula

$$
F(E)=E /(E+U)^{3}
$$

with the surface binding energy $U=7 \mathrm{eV}$ and $g(\alpha)$ are angular distributions

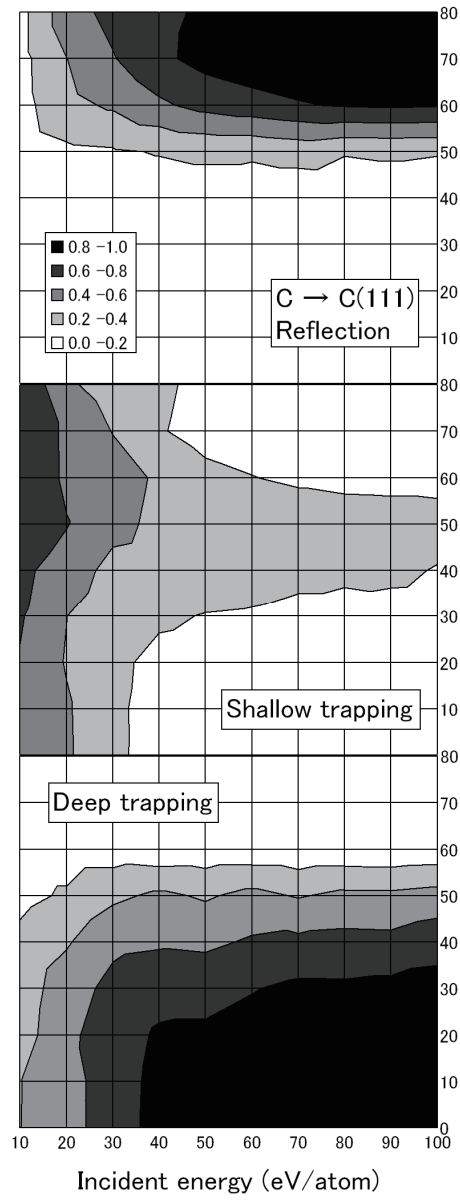

Fig. 6. Reflection and trapping ratio as function of deposition energy and polar incident angle.

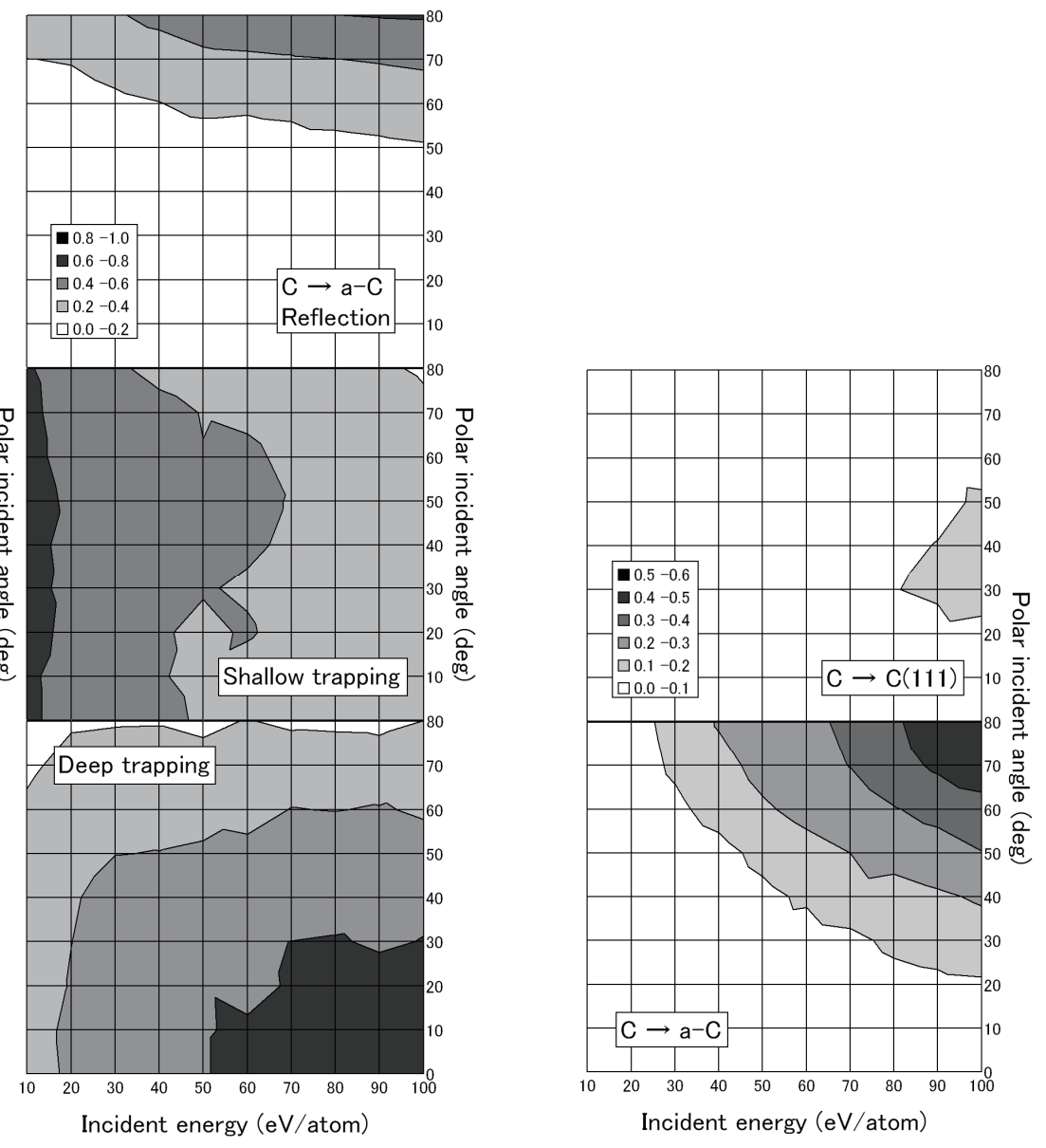

Fig. 7. Sputtering yield as function of deposition energy and polar incident angle. 
Table 1. Redeposition rate on a-C surface.

\begin{tabular}{|c|c|c|c|c|c|c|}
\hline \multirow[b]{2}{*}{ Deposition energy } & \multirow{2}{*}{\multicolumn{2}{|c|}{$\begin{array}{l}\text { This MD } \\
\leq 100 \mathrm{eV}\end{array}$}} & & \multirow{3}{*}{$\begin{array}{c}\text { TBMD }^{9)} \\
100 \mathrm{eV}\end{array}$} & \multicolumn{2}{|c|}{ Experiment $^{23)}$} \\
\hline & & & & & $300 \mathrm{eV}$ & $600 \mathrm{eV}$ \\
\hline Velocity distribution & Normal & Cosine & Isotropic & & & \\
\hline Self-sputtering & 0.06 & 0.18 & 0.25 & 0.43 & $0.47-0.58$ & $0.74-0.79$ \\
\hline Deposition rate & 0.94 & 0.82 & 0.75 & 0.57 & $0.53-0.42$ & $0.26-0.21$ \\
\hline
\end{tabular}

$$
g(\alpha)=\left\{\begin{array}{c}
\delta(\alpha) \\
\cos (\alpha) \\
1
\end{array} .\right.
$$

We considered the normal incidence, the cosine and the isotropic distribution. Table 1 shows the deposition rate on the a-C surface. Other experimental ${ }^{23)}$ and MD ${ }^{9)}$ results are also listed. Since the reflection rate and the sputtering yield increases about grazing incidence, introducing the incident angle distribution decreases the deposition rate. The present result is higher than the other experimental and MD results, because the most of sputtered carbon particles has the deposition energy less than $10 \mathrm{eV}$, which is lower than that of the other cases. Moreover Cooper et al. ${ }^{9)}$ used the relaxing a-C surface but we used the well-relaxed a-C surface for every deposition.

\section{Conclusions}

MD simulations are performed in order to estimate the sputtering yield by low-energy Xe bombardment and the redeposition of sputtered carbon particles.

For the normal incidence of Xe, the MD result of sputtering yield almost agrees with the experimental results. However, the experimental result shows less incident angle dependence than the MD result because it performed on a rough surface. The differential sputtering yield by the low-energy Xe bombardment depends on the incident angle. Sputtered particles by low-energy ion bombardment have the memory of the ion because they go through only a few collisions.

For the redeposition on a-C surface, the reflection and the deep trapping are suppressed. The mean deposition rate with $E$ $\leq 100 \mathrm{eV}$ is $0.82 \sim 0.75$ taking account of incident angle distributions.

\section{Acknowledgments}

This work is partly supported by the engineering digital innovation center and the institute of space and astronautical science of Japan aerospace exploration agency. We would like to thank M. Nakano and I. Funaki for useful discussions.

\section{References}

1) Nakano, M., Kajimura, Y. and Funaki, I.: JAXA Research and Development Report JAXA-RR-09-004 (2009), pp. 1-9 (in Japanese).

2) Muramoto, T., Hyakutake, T., Nishida, M. and Kenmotsu, T.: Proceedings of Space Transpotation Simposium, STEP-2008-17, 2009 (in Japanese).

3) Muramoto, T., Hyakutake, T., Nishida, M. and Kenmotsu, T.: Nucl. Instr. Meth. B 269 (2011), 1752-1754.

4) Smith, R.: Atomic and Ion Collisions in Solids and at Surfaces: Theory, Simulation and Applications, Cambridge University Press, 1997.

5) Juslin, N., Erhart, P., Traskelin, P., Nord, J., Henriksson, K.O.E., Nordlund, K., Salonen, E. and Albe, K.: J. Appl. Phys. 98 (2005), 123520.1-123520.12.

6) Molière, G.: Z. Naturforsch A 2 (1947), p.133-145.

7) Lennard, J.E. and Jones, I.: Proc. R. Soc. London A 106 (1924), 441-477.

8) Thompson, M.W.: Phil. Mag. 18 (1968), 377-414.

9) Cooper, N.C., Fagan, M.S., Goringe, C.M., Marks, N.A. and McKenzie, D.R.: J. Phys. Condens. Matter 14 (2002), 723-730.

10) Beeler, J.R.Jr.: Defects in Solids 13, North-Holland, Amsterdam, 1983.

11) Berendsen, H.J.C., Postma, J.P.M., van Gunsteren, W.F., DiNola, A. and Haak, J.R.: J. Chem. Phys. 81 (1984), 3684-3690.

12) Takeuchi, W. and Yamamura, Y.: Radiat. Eff. 71 (1983), 53-64.

13) Biersack, J.P. and Eckstein, W.: Appl. Phys. A 34 (1984), 73-94.

14) Eckstein, W., Garcia-Rosales, C., Roth, J. and Ottenberger, W.: Max-Plank-Institut fuer Plasmaphysik Report IPP9/82 (1993), p.52.

15) Williams, J.D., Johnson, M.L., and Williams, D.D.: AIAA-2004-3788, 2004.

16) Deltschew, R., Tartz, M., Plicht, V., Hartmann, E., Neumann, H., Leiter, H.J. and Esch, J.: IEPC-01-118, 2001.

17) Doerner, R.P., Whyte, D.G. and Goebel, D.M.: J. Appl. Phys. 93 (2003), 5816-5823.

18) Funaki, I., Nishiyama, K., Kuninaka, H., Toki, K., Shimizu, Y. and Toki, H.: IEPC-01-103, 2001.

19) Gruber, J.R.: IEPC-01-307, 2001.

20) Rosenberg, D. and Wehner, G.K.: J. Appl. Phys. 33 (1962), 1842-1845.

21) Muramoto, T. and Kenmotsu, T.: Nucl. Instrm. Meth. B 255 (2007), 214-218.

22) Kenmotsu, T., Wada, M., Hyakutake, T., Muramoto, T. and Nishida, M.: JAXA Research and Development Report JAXA-RR-09-004 (2009), pp. 26-31 (in Japanese).

23) Miyazawa, T., Misawa, S., Yoshida, S. and Gonda, S.: J. Appl. Phys. 55 (1984), 188-193. 